\title{
O uso de extrato de alho como fitoterapia no controle da hipertensão: uma revisão integrativa
}

The use of garlic extract as herbal medicine in the control of hypertension: an integrative review El uso del extracto de ajo como medicina herbal en el control de la hipertensión: una revisión integradora

Chrisllayne Oliveira da Silva

ORCID: https://orcid.org/0000-0002-0844-0268 Centro Universitário de Ciências e Tecnologia do Maranhão, Brasil

E-mail: chris-layne10@hotmail.com

Wenderson Costa da Silva

ORCID: https://orcid.org/0000-0001-6031-9775 Centro Universitário de Ciências e Tecnologia do Maranhão, Brasil E-mail: wendersoncosta09@hotmail.com

Karine Costa Melo

ORCID: https://orcid.org/0000-0001-8253-859X Centro Universitário de Ciências e Tecnologia do Maranhão, Brasil E-mail:karinemelo09@gmail.com

Alanna Nunes Soares

ORCID: https://orcid.org/0000-0002-0904-4515

Centro Universitário de Ciências e Tecnologia do Maranhão, Brasil

E-mail: alanna_ns@hotmail.com

Linccon Fricks Hernandes

ORCID: https://orcid.org/0000-0002-7642-3080 Escola Superior de Ciências da Santa Casa de Misericórdia de Vitória, Brasil

E-mail: fricksjr@hotmail.com

Rafael Andrade da Silva

ORCID: https://orcid.org/0000-0002-0357-8102 Centro Universitário de Ciências e Tecnologia do Maranhão, Brasil

E-mail: rafael98enfermeiro@gmail.com

Ianeska Bárbara Ribeiro do Nascimento

ORCID: https://orcid.org/0000-0002-5319-289X Centro Universitário de Ciências e Tecnologia do Maranhão, Brasil

E-mail: ianeska@outlook.com

Jayne Oliveira Chaves

ORCID: https://orcid.org/0000-0002-8610-9984 Centro Universitário de Ciências e Tecnologia do Maranhão, Brasil

E-mail: jayneoliveirachaves@gmail.com

Francisco das Chagas Araújo Sousa

ORCID: https://orcid.org/0000-0001-8086-2150 Universidade Estadual do Piauí, Brasil E-mail: chicaovet@gmail.com

Apolo Kassio Barros da Silva

ORCID: https://orcid.org/0000-0003-0526-6169 Centro Universitário de Ciências e Tecnologia do Maranhão, Brasil E-mail: apolo19972009@gmail.com

Ismael Pereira da Silva

ORCID: https://orcid.org/0000-0002-5227-8368

Faculdade de Rondonópolis, Brasil

E-mail: ipereira1090@gmail.com

Carla Vieira Araújo

ORCID: https://orcid.org/0000-0001-5433-6165

Universidade Estadual do Maranhão, Brasil

E-mail: carlavieiral140@gmail.com

Alisson da Silva Alves

ORCID: https://orcid.org/0000-0002-6059-0167 Centro Universitário de Ciências e Tecnologia do Maranhão, Brasil E-mail: alissonalvesfisio@gmail.com

Rayanne Gonçalves Rodrigues Corrêa

ORCID: https://orcid.org/0000-0003-0726-9101

Centro Universitário de Ciências e Tecnologia do Maranhão, Brasil E-mail: rayanneg.r@live.com 


\title{
Resumo
}

O alho age na hipertensão de diversas formas, primeiramente exercendo um efeito dilatador, tanto pela liberação de óxido nítrico, quanto pelo aumento da adenosina disponível, contribuindo dessa forma para o mecanismo hipotensor, podendo-se considerar o alho um forte aliado no tratamento desta patologia, o estudo teve como problemática "Qual a eficácia do uso de extrato de alho como fitoterapia no controle da hipertensão de acordo com a Literatura?”. Onde objetivou-se verificar nas produções cientificas disponíveis os efeitos do extrato de alho em indivíduos hipertensos. O presente estudo trata-se de uma pesquisa bibliográfica do tipo Revisão Integrativa (RI) da literatura, consultou-se por meio de descritores e palavras-chave as bases de dados PubMed, BVS, coordenada pela BIREME e composta de bases de dados bibliográficas produzidas pela Rede BVS, como LILACS, além da base de dados Medline e CINAHL. Ao final oito (8) artigos atenderam a questão norteadora e foram adicionados ao estudo e evidenciou o uso do alho na prática clínica, os beneficios da utilização do alho como terapia complementar no tratamento HAS e dentro da dieta, além dos principais efeitos colaterais. Conclui-se que aumentar a ingestão de vegetais allium, pode estar associada a redução de triglicerídeos e depuração de creatinina, o que proporciona uma melhoria da função renal e diminuição da pressão arterial.

Palavras-chave: Patologia; Allium; Terapêutica.

\begin{abstract}
Garlic acts on hypertension in several ways, first, exerting a dilating effect, both by the release of nitric oxide and by the increase in available adenosine, thus contributing to the hypotensive mechanism, and garlic can be considered a strong ally in the treatment of this pathology, the study had as a problem "effectiveness of using garlic extract as herbal medicine in controlling hypertension according to the literature?". Where the objective was to verify in the available scientific productions the effects of garlic extract in hypertensive individuals. The present study is a bibliographic search of the type Integrative Review (IR) of the literature, the PubMed databases were consulted through descriptors and keywords, BVS, coordinated by BIREME and composed of bibliographic databases produced by the BVS Network, such as LILACS, in addition to the Medline database and CINAHL. At the end, eight (8) articles answered the guiding question and were added to the study and highlighted the use of garlic in clinical practice, the benefits of using garlic as a complementary therapy in the treatment of hypertension and within the diet, in addition to the main side effects. It was concluded that increasing the intake of allium vegetables may be associated with a reduction in triglycerides and creatinine clearance, which provides an improvement in renal function and a decrease in blood pressure.
\end{abstract}

Keywords: Pathology; Allium; Therapeutics.

\section{Resumen}

El ajo actúa sobre la hipertensión de varías formas, en primer lugar, al ejercer un efecto dilatador, tanto por la liberación de óxido nítrico como por el aumento de la adenosina disponible, contribuyendo así al mecanismo hipotensor, y el ajo puede considerarse un fuerte aliado en el tratamiento de esta patología, el estudio tuvo como problema "¿Cuál es el eficacia del uso de extracto de ajo como medicina herbal en el control de la hipertensión según la literatura?”. Donde el objetivo fue verificar en las producciones científicas disponibles los efectos del extracto de ajo en hipertensos. El presente estudio es una búsqueda bibliográfica del tipo Revisión Integrativa (RI) de la literatura, se consultaron las bases de datos PubMed mediante descriptores y palabras clave, BVS, coordinada por BIREME y compuesta por bases de datos bibliográficas producidas por la Red BVS, como LILACS, además de la base de datos Medline y CINAHL. Al final, ocho (8) artículos respondieron a la pregunta orientadora y se agregaron al estudio y destacaron el uso del ajo en la práctica clínica, los beneficios del uso del ajo como terapia complementaria en el tratamiento de la HSA y dentro de la dieta, además de los principales efectos secundarios. Se concluyó que el aumento de la ingesta de vegetales allium puede estar asociado con una reducción de los triglicéridos y el aclaramiento de creatinina, lo que proporciona una mejora de la función renal y una disminución de la presión arterial.

Palabras clave: Patologia; Alliu; Terapéutica.

\section{Introdução}

Atualmente o Sistema Único de Saúde (SUS) vem se mostrando favorável a utilização de recursos terapêuticos que sejam mais eficazes em muitas das instâncias de tratamento e economicamente mais acessíveis, principalmente, no que se refere às Práticas Integrativas e Complementares (PIC). A medicina tradicional e complementar, além de promoverem a redução dos custos, têm se mostrado eficazes na promoção da saúde, evitando que a doença se instale e que suas consequências sejam muito graves (Ischkanian \& Pelicioni, 2012). De acordo com Fernández-Cervilla (2013), as terapias de base biológica são aquelas que utilizam produtos naturais, tais como ervas dietéticas e plantas medicinais frescas ou secas, alimentos e vitaminas para o manejo de doenças. 
A fitoterapia é uma terapêutica caracterizada pela utilização de plantas medicinais em suas diferentes formas farmacêuticas, sem o uso de substâncias ativas isoladas, ainda que sejam de origem vegetal. O uso das plantas medicinais na arte de curar é uma forma de tratamento de origem muito antiga, relacionada aos primórdios da medicina e fundamentada nas informações que perduraram através de gerações. Ao longo da história os produtos de origem vegetal formaram as bases para o tratamento de diferentes doenças (Ministério da Saúde, 2015).

Segundo a Organização Mundial da Saúde (OMS) os fitoterápicos são produtos e materiais à base de plantas e ervas. O termo "erva", inclui material de planta em seu estado bruto, tais como folhas, flores, frutos, sementes, fragmentados ou em pó e processados por métodos locais e utilizados de forma tradicional. A fitoterapia, associada ou não ao tratamento convencional, traz inúmeros benefícios e uma dimensão mais humanizada e integral do paciente, resgata sua condição histórico-cultural, e contribui para a valorização do indivíduo como agente de sua própria história (Bueno, Martínez, \& Bueno, 2016).

Ultimamente, tem se observado o aumento da expectativa de vida da população e com isso observa-se também o aumento da prevalência de patologias como a Hipertensão Arterial Sistêmica (HAS), Diabetes Mellitus (DM) e dislipidemias, doenças essas que provocam grande impacto na qualidade de vida e hábitos da população. A procura por tratamentos alternativos ou adicionais ao farmacológico vem crescendo significativamente, e o uso de chás e plantas medicinais se torna cada vez mais frequente (Silva \& Hahn, 2011).

A HAS é fator de risco para o desenvolvimento de Doenças Cardiovasculares (DCV) e síndrome com manifestações próprias e características típicas. A hipertensão está notoriamente associada a diversas complicações, como Acidente Vascular Encefálico (AVE), Doença Arterial Periférica (DAP), Insuficiência Cardíaca (IC), Doença Renal Crônica (DRC), Infarto Agudo do Miocárdio (IAM) e Doença Arterial Coronariana (DAC) (Nobre, Coelho, Lopes, \& Geleilete, 2013).

A literatura tem demostrado os efeitos positivos do alho sobre a HAS. Chagas, Zanetti e Donatini (2012) escrevem que o alho age na hipertensão de diversas formas, exercendo um efeito dilatador, tanto pela liberação de óxido nítrico, quanto pelo aumento da adenosina disponível, contribuindo dessa forma para o mecanismo hipotensor, podendo-se considerar o alho um forte aliado no tratamento desta patologia.

Diante do exposto apresenta-se a necessidade de avaliar a o uso de extrato de alho como fitoterapia no controle da hipertensão, para tal o estudo teve como problemática "Qual a eficácia do uso de extrato de alho como fitoterapia no controle da hipertensão de acordo com a Literatura?”. Onde objetivou-se verificar nas produções cientificas disponíveis os efeitos do extrato de alho em indivíduos hipertensos, mais especificamente, analisar o efeito do alho como tratamento anti-hipertensivo; explicar os benefícios do allium vegetais na dieta; descrever a utilização do alho na redução das morbidades e mortalidades; e identificar efeitos colaterais do uso de alho. Dessa forma, a relevância da pesquisa foi apresentar evidências cientificas presentes na literatura acerca da utilização do extrato de alho como fitoterapia no controle da HAS, descrevendo os principais benefícios da sua aplicação na rotina e elencando pontos positivos e negativos.

\section{Metodologia}

O presente estudo trata-se de uma pesquisa bibliográfica do tipo Revisão Integrativa (RI) da literatura. Este procedimento foi escolhido por possibilitar a síntese e análise do conhecimento científico já produzido sobre o tema "O Uso De Extrato De Alho Como Fitoterapia No Controle Da Hipertensão: Uma Revisão Integrativa”. Esta revisão utilizou a metodologia proposta no estudo de Oliveira et al. (2016).

De acordo com Souza, Silva e Carvalho (2010), a RI tem sido vista como um instrumento ímpar no campo das ciências da saúde, pois sintetiza os estudos disponíveis sobre determinado tema e direciona a prática respaldando-se em conhecimento científico. Apesar de que combinar dados de delineamento de pesquisa diversos seja complicado e difícil, conduzir uma revisão integrativa a partir da inclusão de uma organizada e rigorosa abordagem do processo, sobretudo da análise de dados, tem como 
resultado a diminuição de vieses e erros. Portanto, é imperativo instituir a RI como dispositivo válido da Prática Baseada em Evidências (PBE).

O tema determinou a construção da estratégia PICO, que representa um acrônimo para Paciente (P), Intervenção (I), Comparação (C) e Desfechos (O-outcomes), na qual foi utilizada como questão norteadora desta revisão integrativa da literatura: “Quais evidências científicas recomendam para o controle da pressão arterial o uso de extrato de alho em indivíduos hipertensos?".

Para a localização dos estudos relevantes, que respondessem à pergunta de pesquisa, utilizou-se de descritores indexados e não indexados (palavras-chave) nos idiomas português, inglês e espanhol. Os descritores foram obtidos a partir do Medical Subject Headings (MESH), dos Descritores em Ciências da Saúde (DeCS) e dos títulos CINAHL, como mostra o Quadro 1.

Consultou-se por meio de descritores e palavras-chave as bases de dados PubMed da National Library of Medicine; BVS (Biblioteca Virtual da Saúde), coordenada pela BIREME e composta de bases de dados bibliográficas produzidas pela Rede BVS, como LILACS, além da base de dados Medline e outros tipos de fontes de informação e CINAHL (Cumulative Index to Nursing and Allied Health Literature).

Quadro 1. Elementos da estratégia PICO, descritores e palavras-chave utilizados. Caxias, MA, Brasil, 2019.

\begin{tabular}{|c|c|c|c|c|c|}
\hline & Elementos & Mesh & Decs & Títulos Cinahl & Palavras-chave \\
\hline $\mathrm{P}$ & "Hipertensão" & "Hypertension" & $\begin{array}{l}\text { "Hypertension” } \\
\text { "Hipertensión" } \\
\text { "Hipertensão", }\end{array}$ & "Hypertension" & $\begin{array}{l}\text { "Hypertension” } \\
\text { "Hipertensión" } \\
\text { "Hipertensão", }\end{array}$ \\
\hline I & "Alho" & $\begin{array}{c}\text { "Garlic" } \\
\text { "Plant extracts" }\end{array}$ & $\begin{array}{c}\text { "Garlic" } \\
\text { "Ajo" } \\
\text { "Alho" } \\
\text { "Plant extracts" } \\
\text { "Extractos } \\
\text { vegetales" } \\
\text { "Extratos } \\
\text { vegetais" }\end{array}$ & $\begin{array}{c}\text { "Garlic" } \\
\text { "Plant extracts" }\end{array}$ & $\begin{array}{c}\text { "Garlic" } \\
\text { "Ajo" } \\
\text { "Alho" } \\
\text { "Plant extracts" } \\
\text { "Extractos } \\
\text { vegetales" } \\
\text { "Extratos } \\
\text { vegetais" }\end{array}$ \\
\hline $\mathrm{C}$ & - & - & - & - & - \\
\hline $\mathrm{O}$ & "Normotensão" & - & $\begin{array}{l}\text { "Control", } \\
\text { "Control", } \\
\text { "Controle", }\end{array}$ & - & $\begin{array}{l}\text { "Control", } \\
\text { "Control", } \\
\text { "Controle", }\end{array}$ \\
\hline
\end{tabular}

Fonte: Descritores, Títulos e Palavras-chaves (2019).

Ressalta-se que o elemento C da estratégia PICO não foi abordado nesta pesquisa, pois esta não tem por objetivo comparar intervenções. Os termos utilizados durante a pesquisa foram classificados e combinados nos bancos de dados, resultando em estratégias específicas de cada base, como mostra o Quadro 2. 
Quadro 2. Estratégias de busca utilizadas nas bases de dados BIREME, PUBMED e CINAHL. Caxias, MA, Brasil, 2019.

\begin{tabular}{|c|c|c|c|c|}
\hline $\begin{array}{l}\text { BASE DE } \\
\text { DADOS }\end{array}$ & ESTRATÉGIA DE BUSCA & 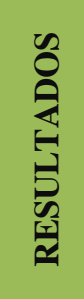 & 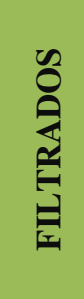 & 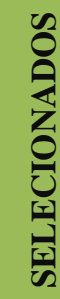 \\
\hline $\begin{array}{c}\text { BIREME } \\
\text { (descritores Decs) }\end{array}$ & $\begin{array}{l}\text { tw:(tw:((tw:(hipertensão )) AND (tw:(alho OR extratos vegetais )) } \\
\text { AND (tw:(controle ))) AND (instance:"regional") AND } \\
\text { ( fulltext:("1"))) AND (instance:"regional") }\end{array}$ & 416 & 264 & 3 \\
\hline $\begin{array}{l}\text { PubMed } \\
\text { (descriptors } \\
\text { MeSH) }\end{array}$ & $\begin{array}{l}\text { (("hypertension"[MeSH Terms] OR "hypertension"[All Fields]) } \\
\text { AND (("garlic"[MeSH Terms] OR "garlic"[All Fields]) OR ("plant } \\
\text { extracts"[MeSH Terms] OR ("plant"[All Fields] AND "extracts"[All } \\
\text { Fields]) OR "plant extracts"[All Fields]))) AND ("prevention and } \\
\text { control"[Subheading] OR ("prevention"[All Fields] AND } \\
\text { "control"[All Fields]) OR "prevention and control"[All Fields] OR } \\
\text { "control"[All Fields] OR "control groups"[MeSH Terms] OR } \\
\text { ("control"[All Fields] AND "groups"[All Fields]) OR "control } \\
\text { groups"[All Fields]) }\end{array}$ & 793 & 163 & 3 \\
\hline $\begin{array}{l}\text { CINAHL } \\
\text { (CINAHL } \\
\text { Headings) }\end{array}$ & Hypertension AND (Garlic or Plant extracts) AND Control & 299 & 34 & 2 \\
\hline
\end{tabular}

Fonte: Bases de dados.

Como critérios de inclusão utilizaram-se estudos disponíveis em sua totalidade, publicados nos últimos dez anos, de 2009 até 2019, nos idiomas Português, Espanhol e Inglês. Foram excluídos da busca inicial capítulos de livros, resumos, textos incompletos, teses, dissertações, monografias, relatos técnicos e outras formas de publicação que não fossem artigos científicos completos.

A análise para seleção dos estudos foi realizada em duas fases, a saber: na primeira, os estudos foram pré-selecionados segundo os critérios de inclusão e exclusão e de acordo com a estratégia de funcionamento e busca de cada base de dados. Durante a análise e interpretação dos resultados, as informações coletadas nos artigos científicos foram analisadas e criaram-se categorias analíticas que facilitaram a ordenação e a sumarização de cada estudo. Essa categorização foi realizada de forma descritiva, indicando os dados mais relevantes para o estudo.

$\mathrm{Na}$ segunda fase os estudos foram analisados quanto ao potencial de participação no estudo, avaliando o atendimento à questão de pesquisa, bem como o tipo de investigação, objetivos, amostra, método, desfechos e conclusão, resultando em oito (8) artigos. Ao final de toda análise, oito (8) artigos atenderam a questão norteadora e foram adicionados ao estudo.

O estudo levou em consideração os aspectos éticos da pesquisa quanto às citações dos estudos, respeitando a autoria das ideias, os conceitos e as definições presentes nos artigos incluídos na revisão.

As evidências científicas foram classificadas segundo os níveis e graus de recomendação propostos por Bork (2011), onde são apresentados sete níveis de evidência: nível 1, Revisão Sistemática; nível 2, Ensaio Clínico Randomizado; nível 3, Coorte; nível 4, Caso Controle; nível 5, Série de Casos; nível 6, Opiniões de Especialistas; e nível 7, Estudos Pré-Clínicos (animais/in vitro). Além de três graus de recomendação: grau A, onde o resultado recomenta a intervenção; grau B, onde o resultado não é conclusivo ou não é o suficiente para confirmar a hipótese e; grau C, onde o resultado contraindica a intervenção. 


\section{Resultados e Discussão}

Encontraram-se quatrocentos e dezesseis (416) estudos como busca geral na BVS, sendo que limitando a busca para artigos com texto completo, realizado com humanos e nos últimos dez anos, obteve-se duzentos e sessenta e quatro (264) estudos, destes foram analisados títulos e resumos onde apenas três (3) estudos foram condizentes com a questão desta pesquisa.

Na base PUBMED, como busca total foram encontrados setecentos e noventa e três (793) estudos, aplicando na pesquisa o filtro que limita por texto completo dos últimos dez anos, com humanos, obteve-se cento e sessenta e três (163) estudos, destes foram analisados títulos e resumos e teve como resultado final de três (3) estudos.

$\mathrm{Na}$ CINAHL foram obtidos duzentos e noventa e nove (299) estudos como busca geral, sendo que limitando a busca para artigos com texto completo realizados nos últimos dez anos com humanos, obteve-se trinta e quatro (34) estudos, sendo três (3) condizentes com a questão desta pesquisa após a análise dos títulos e resumos.

O Quadro 3 se refere aos autores das bases teóricas utilizados no estudo, de forma a evidenciar de que trata os artigos científicos; o autor do artigo, assim como o tipo de pesquisa, o objetivo e os resultados a que se refere cada publicação utilizada na elaboração do presente estudo.

Quadro 3. Distribuição das publicações cientificas com autor, ano, objetivos, tipo de estudo e resultados. Caxias, MA, Brasil, 2019.

\begin{tabular}{|c|c|c|c|c|}
\hline Autores & Objetivo principal & Perfil amostral & $\begin{array}{c}\text { Intervenções/ } \\
\text { Interesse }\end{array}$ & Principais resultados \\
\hline $\begin{array}{c}\text { Stabler, Tejani, } \\
\text { Huynh e Fowkes } \\
\text { (2012) }\end{array}$ & $\begin{array}{l}\text { Determinar se o uso de alho } \\
\text { como monoterapia, em } \\
\text { pacientes hipertensos, } \\
\text { diminui o risco de } \\
\text { morbidade e mortalidade } \\
\text { cardiovascular em } \\
\text { comparação ao placebo. }\end{array}$ & $\begin{array}{l}\text { Ensaios clínicos } \\
\text { randomizados } \\
\text { totalizando dois } \\
\text { artigos. }\end{array}$ & $\begin{array}{l}\text { Realizar uma } \\
\text { revisão baseada em } \\
\text { evidências da } \\
\text { literatura sobre o } \\
\text { uso do alho como } \\
\text { monoterapia em } \\
\text { pacientes } \\
\text { hipertensos. }\end{array}$ & $\begin{array}{l}\text { Ingerir } 200 \mathrm{mg} \text { de alho em pó } \\
\text { administrados três vezes ao dia, } \\
\text { além da hidroterapia com } \\
\text { hidroclorotiazida e triantereno, } \\
\text { produziram uma redução média da } \\
\text { pressão arterial sistólica e } \\
\text { diastólica versus placebo. }\end{array}$ \\
\hline $\begin{array}{c}\text { Simons, } \\
\text { Wollersheim e } \\
\text { Thien (2009) }\end{array}$ & $\begin{array}{l}\text { Realizar uma avaliação da } \\
\text { qualidade dos estudos } \\
\text { sobre a utilização do alho } \\
\text { na redução da pressão } \\
\text { arterial. }\end{array}$ & $\begin{array}{l}\text { Meta-análise } \\
\text { totalizando } \\
\text { trinta e dois } \\
\text { ensaios clínicos } \\
\text { randomizados. }\end{array}$ & $\begin{array}{l}\text { Realizar uma } \\
\text { revisão baseada em } \\
\text { evidências da } \\
\text { literatura sobre a } \\
\text { qualidade dos } \\
\text { estudos referentes à } \\
\text { utilização do alho na } \\
\text { redução da pressão } \\
\text { arterial. }\end{array}$ & $\begin{array}{l}\text { A qualidade metodológica dos } \\
\text { estudos foi pobre, apenas quatro } \\
\text { estudos tiveram ocultação de } \\
\text { alocação adequada. Além disso, } \\
\text { metade dos estudos não relatou } \\
\text { dados sobre a medida da pressão } \\
\text { arterial. }\end{array}$ \\
\hline $\begin{array}{l}\text { Sobenin, } \\
\text { Andrianova, } \\
\text { Fomchenkov, } \\
\text { Gorchakova e } \\
\text { Orekhov (2009) }\end{array}$ & $\begin{array}{l}\text { Comparar o uso de pílulas } \\
\text { de alho em pó em relação } \\
\text { ao uso de pílulas de alho } \\
\text { comuns para reduzir a } \\
\text { pressão arterial. }\end{array}$ & $\begin{array}{l}\text { Um total de } 84 \\
\text { homens (35- } 70 \\
\text { anos) } \\
\text { diagnosticados } \\
\text { com hipertensão } \\
\text { leve ou } \\
\text { moderada. }\end{array}$ & $\begin{array}{l}\text { Ingerir pílulas } \text { de } \\
\text { alho em pó } \\
\text { pílulas de } \\
\text { comum } \\
\begin{array}{ll}\text { comparação } & \text { em } \\
\text { placebo. } & \text { ao }\end{array} \\
\end{array}$ & $\begin{array}{l}\text { A ingestão de pílulas de alho em } \\
\text { pó ou pílulas de alho comum } \\
\text { mostrou diminuição da pressão } \\
\text { arterial e seus resultados não } \\
\text { diferiram significativamente, já } \\
\text { os placebos não mostraram } \\
\text { eficácia. }\end{array}$ \\
\hline $\begin{array}{l}\text { Wang, J. } \\
\text { Yang, Qin e } \\
\text { X.-J. Yang } \\
\quad(2015)\end{array}$ & $\begin{array}{l}\text { Investigar o efeito do uso } \\
\text { de alho na pressão arterial. }\end{array}$ & $\begin{array}{l}\text { Meta-análise } \\
\text { totalizando } \\
\text { dezessete } \\
\text { ensaios clínicos } \\
\text { randomizados. }\end{array}$ & $\begin{array}{l}\text { Realizar uma } \\
\text { revisão baseada em } \\
\text { evidências da } \\
\text { literatura sobre o } \\
\text { efeito do uso de alho } \\
\text { na pressão arterial. }\end{array}$ & $\begin{array}{l}\text { Os resultados mostraram que o } \\
\text { alho tem um efeito } \\
\text { significativamente maior na } \\
\text { redução da pressão arterial em } \\
\text { comparação ao placebo. }\end{array}$ \\
\hline
\end{tabular}




\begin{tabular}{|c|c|c|c|c|}
\hline $\begin{array}{l}\text { Ried, Frank e } \\
\text { Stocks (2013) }\end{array}$ & $\begin{array}{l}\text { Avaliar o efeito, dose- } \\
\text { resposta, tolerabilidade e } \\
\text { aceitabilidade de diferentes } \\
\text { doses de extrato de alho } \\
\text { envelhecido como um } \\
\text { tratamento adjunto à } \\
\text { medicação anti- } \\
\text { hipertensiva existente em } \\
\text { pacientes com hipertensão } \\
\text { não controlada. }\end{array}$ & $\begin{array}{l}\text { Um total de } 79 \\
\text { participantes } \\
\text { diagnosticados } \\
\text { com hipertensão } \\
\text { arterial não } \\
\text { controlada. }\end{array}$ & $\begin{array}{l}\text { Consumir extrato de } \\
\text { alho envelhecido } \\
\text { em comparação ao } \\
\text { placebo. }\end{array}$ & $\begin{array}{l}\text { O consumo do extrato de alho } \\
\text { envelhecido é superior ao placebo } \\
\text { na redução da pressão arterial em } \\
\text { pacientes com hipertensão não } \\
\text { controlada, seu uso foi bem } \\
\text { tolerado e altamente aceitável, } \\
\text { porém, embora raros, distúrbios } \\
\text { gastrointestinais foram relatados. }\end{array}$ \\
\hline $\begin{array}{l}\text { Ried, Frank e } \\
\text { Stocks (2010) }\end{array}$ & $\begin{array}{l}\text { Avaliar o efeito, } \\
\text { tolerabilidade } \\
\text { aceitabilidade do extrato de } \\
\text { alho envelhecido como um } \\
\text { tratamento adjunto à } \\
\text { medicação anti- } \\
\text { hipertensiva tratada, mas } \\
\text { não controlada. }\end{array}$ & $\begin{array}{l}\text { Um total de } 25 \\
\text { participantes } \\
\text { com hipertensão } \\
\text { tratada, porém } \\
\text { não controlada. }\end{array}$ & $\begin{array}{l}\text { Ingerir cápsulas de } \\
\text { alho envelhecido } \\
\text { em comparação ao } \\
\text { placebo. }\end{array}$ & $\begin{array}{l}\text { A ingestão das cápsulas de alho } \\
\text { envelhecido é superior ao placebo } \\
\text { na redução da pressão arterial } \\
\text { sistólica em pacientes com } \\
\text { hipertensão tratada, mas não } \\
\text { controlada. O extrato de alho } \\
\text { envelhecido foi geralmente bem } \\
\text { tolerado e o nível de redução da } \\
\text { pressão arterial obtido foi } \\
\text { comparável ao de medicação anti- } \\
\text { hipertensiva comum. }\end{array}$ \\
\hline $\begin{array}{l}\text { Bahadoran, } \\
\text { Mirmiran, } \\
\text { Momenan e } \\
\text { Azizi (2017) }\end{array}$ & $\begin{array}{l}\text { Investigar a associação } \\
\text { entre o consumo habitual } \\
\text { de vegetais de alho (alho e } \\
\text { cebola) e a incidência de } \\
\text { desfechos de doença } \\
\text { cardiovascular, } \\
\text { hipertensão, doença renal } \\
\text { crônica e diabetes tipo } 2 \text {. }\end{array}$ & $\begin{array}{lr}\begin{array}{l}\text { Homens } \\
\text { mulheres }\end{array} \\
\text { adultos, } \\
\text { participantes } & \text { do } \\
\text { Estudo } & \text { de } \\
\text { Lipídios } & \text { e } \\
\text { Glucose } & \text { de } \\
\text { Teerã } & (2006- \\
2008 \text { a } & 2012- \\
2014), & \text { foram } \\
\text { recrutados. }\end{array}$ & $\begin{array}{l}\text { Consumir alho e } \\
\text { cebola de acordo } \\
\text { com os hábitos } \\
\text { alimentares } \\
\text { individuais de cada } \\
\text { participante. }\end{array}$ & $\begin{array}{l}\text { A maior ingestão habitual de } \\
\text { legumes allium foi associada a um } \\
\text { risco reduzido de } 64 \% \text { de } \\
\text { desfechos } \\
\text { cardiovasculares, menor } \\
\text { incidência de doença renal } \\
\text { crônica, e diminuiu o } \\
\text { desenvolvimento da hipertensão, } \\
\text { porém, não houve nenhuma } \\
\text { associação significativa entre a } \\
\text { ingestão de vegetais de allium e o } \\
\text { risco de diabetes tipo } 2 \text {. }\end{array}$ \\
\hline $\begin{array}{l}\text { Xiong et al. } \\
\text { (2015) }\end{array}$ & $\begin{array}{l}\text { Revisar sistematicamente a } \\
\text { literatura médica para } \\
\text { investigar as evidências } \\
\text { atuais de alho para o } \\
\text { tratamento da hipertensão. }\end{array}$ & $\begin{array}{l}\text { Meta-análise } \\
\text { totalizando sete } \\
\text { ensaios clínicos } \\
\text { randomizados. }\end{array}$ & $\begin{array}{l}\text { Realizar uma } \\
\text { revisão baseada em } \\
\text { evidências da } \\
\text { literatura sobre o } \\
\text { efeito do uso de alho } \\
\text { na hipertensão. }\end{array}$ & $\begin{array}{l}\text { O estudo revelou que o uso do alho } \\
\text { tem um efeito redutor } \\
\text { significativo, tanto na pressão } \\
\text { arterial sistólica quando na pressão } \\
\text { arterial diastólica. }\end{array}$ \\
\hline
\end{tabular}

Fonte: Artigos pesquisados (2019).

É interessante observar que a maioria dos estudos teve como interesse realizar uma revisão baseada em evidências, além disso, quase toda totalidade dos estudos demonstraram resultados positivos quanto ao uso do extrato de alho no controle da HAS, o que favorece a possibilidade de ser utilizado na prática clínica.

O Quadro 4 a seguir refere-se aos autores das bases teóricas utilizados no estudo, de maneira a evidenciar os títulos das pesquisas; o ano de publicação; o país onde o estudo foi realizado; o delineamento da pesquisa; nível de evidência; e o grau de recomendação. 
Quadro 4. Distribuição das publicações incluídas segundo o título, ano de publicação, país onde o estudo foi realizado, delineamento da pesquisa, nível de evidência e grau de recomendação. Caxias, MA, Brasil, 2019.

\begin{tabular}{|c|c|c|c|c|c|c|}
\hline Autores & Título & $\begin{array}{l}\text { Base de dado } \\
\text { /ano de } \\
\text { publicação }\end{array}$ & País & $\begin{array}{l}\text { Delineamento } \\
\text { da pesquisa }\end{array}$ & $\begin{array}{l}\text { Nível de } \\
\text { evidência }\end{array}$ & $\begin{array}{c}\text { Grau de } \\
\text { recomendação }\end{array}$ \\
\hline $\begin{array}{l}\text { Stabler et } \\
\text { al. (2012) }\end{array}$ & $\begin{array}{l}\text { Garlic for the prevention of } \\
\text { cardiovascular morbidity and } \\
\text { mortality in hypertensive patients } \\
\text { (Review) }\end{array}$ & $\begin{array}{l}\text { PUBMED/ } \\
2012\end{array}$ & $\begin{array}{l}\text { Reino } \\
\text { Unido }\end{array}$ & $\begin{array}{c}\text { Revisão } \\
\text { Sistematica }\end{array}$ & 1 & B \\
\hline $\begin{array}{l}\text { Simons et } \\
\text { al. (2009) }\end{array}$ & $\begin{array}{l}\text { A systematic review on the } \\
\text { influence of trial quality on the } \\
\text { effect of garlic on blood pressure }\end{array}$ & $\begin{array}{l}\text { PUBMED/ } \\
2009\end{array}$ & Holanda & $\begin{array}{c}\text { Revisão } \\
\text { Sistemática }\end{array}$ & 1 & $\mathrm{C}$ \\
\hline $\begin{array}{l}\text { Sobenin et } \\
\text { al. (2009) }\end{array}$ & $\begin{array}{l}\text { Time-released garlic powder } \\
\text { tablets lower systolic and diastolic } \\
\text { blood pressure in men with mild } \\
\text { and moderate arterial } \\
\text { hypertension }\end{array}$ & $\begin{array}{l}\text { PUBMED/ } \\
2009\end{array}$ & Rússia & $\begin{array}{l}\text { Ensaio Clinico } \\
\text { Randomizado }\end{array}$ & 2 & $\mathrm{~A}$ \\
\hline $\begin{array}{l}\text { Wang et al. } \\
\text { (2015) }\end{array}$ & $\begin{array}{l}\text { Effect of Garlic on Blood } \\
\text { Pressure: A Meta-Analysis }\end{array}$ & $\begin{array}{l}\text { BIREME/ } \\
2015\end{array}$ & China & $\begin{array}{c}\text { Revisão } \\
\text { Sistemática }\end{array}$ & 1 & $\mathrm{~A}$ \\
\hline $\begin{array}{l}\text { Ried et al. } \\
\text { (2013) }\end{array}$ & $\begin{array}{l}\text { Aged garlic extract reduces blood } \\
\text { pressure in hypertensives: a dose- } \\
\text { response trial }\end{array}$ & $\begin{array}{c}\text { BIREME/ } \\
2013\end{array}$ & Austrália & $\begin{array}{l}\text { Ensaio Clinico } \\
\text { Randomizado }\end{array}$ & 2 & $\mathrm{~A}$ \\
\hline $\begin{array}{l}\text { Ried et al. } \\
\text { (2010) }\end{array}$ & $\begin{array}{l}\text { Aged garlic extract lowers blood } \\
\text { pressure in patients with treated } \\
\text { but uncontrolled hypertension: A } \\
\text { randomised controlled trial }\end{array}$ & $\begin{array}{l}\text { BIREME/ } \\
2010\end{array}$ & Austrália & $\begin{array}{l}\text { Ensaio Clinico } \\
\text { Randomiado }\end{array}$ & 2 & $\mathrm{~A}$ \\
\hline $\begin{array}{l}\text { Bahadoran } \\
\text { et al. } \\
\text { (2017) }\end{array}$ & $\begin{array}{l}\text { Allium vegetable intakes and the } \\
\text { incidence of cardiovascular } \\
\text { disease, hypertension, chronic } \\
\text { kidney disease, and type } 2 \\
\text { diabetes in adults: a longitudinal } \\
\text { follow-up study }\end{array}$ & $\begin{array}{c}\text { CINAHL/ } \\
2017\end{array}$ & Irã & $\begin{array}{l}\text { Ensaio Clinico } \\
\text { Randomizado }\end{array}$ & 2 & A \\
\hline $\begin{array}{l}\text { Xiong et al. } \\
\text { (2015) }\end{array}$ & $\begin{array}{l}\text { Garlic for hypertension: A } \\
\text { systematic review and meta- } \\
\text { analysis of randomized controlled } \\
\text { trials }\end{array}$ & $\begin{array}{l}\text { CINAHL/ } \\
2015\end{array}$ & China & $\begin{array}{c}\text { Revisão } \\
\text { Sistemática }\end{array}$ & 1 & B \\
\hline
\end{tabular}

Fonte: Artigos pesquisados (2019).

Quanto ao nível de evidência observou-se que metade dos delineamentos de pesquisa inclusos neste estudo foram revisões sistemáticas e a outra metade composta de ensaios clínicos randomizados, dessa forma, apresentaram um alto nível de evidência. Além disso, a maioria alcançou grau de recomendação A para prática clínica.

\section{O uso do alho na prática clínica}

O alho é um alimento tradicional utilizado mundialmente, apresenta ainda efeitos terapêuticos em algumas patologias. O alho possui a capacidade de provocar efeitos anti-hipertensivos através da inibição das Enzimas Conversoras de Angiotensina 
(ECAs), minimizando a pressão alta. Os suplementos são compostos por alho em pó, extrato de alho envelhecido e óleo de alho, que apresentam efeitos diferentes sobre a pressão sanguínea (Wang, J. Yang, Qin, \& X.-J. Yang, 2015).

Os suplementos de alho apresentam resultados superiores ao placebo na redução da pressão arterial em pacientes hipertensos, especialmente naqueles com Pressão Arterial Sistólica (PAS) elevada. Conjuntamente o extrato de alho envelhecido apresenta efeito superior ao placebo na redução da PAS em pacientes com hipertensão arterial descompensada. A redução observada na PAS é comparável com a alcançada através de medicamentos anti-hipertensivos que são comumente prescritos, apresentando uma importância clínica (Ried, Frank, \& Stocks, 2010; Wang et al., 2015; Xiong et al., 2015).

Em um estudo randomizado, Sobenin et al. (2009) relataram que um dos suplementos dietéticos à base de alho, o Allicor, produz um efeito hipotensivo estatisticamente significativo tanto na PAS quanto na Pressão Arterial Diastólica (PAD) em indivíduos com hipertensão arterial leve a moderada. Tanto o Allicor quanto o Kwai induziram reduções moderadas na pressão arterial, mas estatisticamente significativas, na PAS. Esses achados mostram que os mecanismos de ação hipotensora das preparações à base de alho podem ser bem diferentes daqueles dos agentes farmacológicos convencionais usados no tratamento da hipertensão arterial e podem ser referidos à regulação biológica complexa da pressão arterial.

\section{Utilização do alho como terapia complementar no tratamento HAS}

O estudo de Wang et al. (2015) relatam que o efeito anti-hipertensivo foi produzido a partir da suplementação de 480mg de alho, e em outro o grupo de hipertensos que fez a suplementação de alho teve a diminuição significativa da pressão arterial com o aumento da dose e da duração da suplementação.

Na pesquisa de Ried et al. (2010) em que um grupo controle e um grupo de pacientes com hipertensão não controlada fizeram o uso do alho, o efeito do tratamento durante 12 semanas foi aparente entre os dois grupos do estudo, em que se obteve diferença média na PAS $\pm \mathrm{EP}:-10,2 \pm 4,3, \mathrm{p}=0,0361$, apesar de o efeito não ter sido perceptível em todos os pacientes.

De acordo com Bahadoran et al. (2017), a ingestão de vegetais allium foi associada a redução considerável dos riscos de desenvolvimento de doenças cardiovasculares, doenças renais e hipertensão arterial, cada $10 \mathrm{~g} /$ semana de aumento de ingestão de vegetais allium, foi associado com a redução de triglicerídeos e depuração de creatinina, favorecendo a melhoria da função renal e diminuição da pressão arterial.

Na revisão sistemática de Xiong et al. (2015) foi relatado que processados de alho em cápsula reduziu significativamente a pressão arterial por uma média de $8,77 \mathrm{mmHg}$, depois de apenas duas semanas $(\mathrm{P}<0,01)$ e uma média de $8,05 \mathrm{mmHg}$ após 8 semanas $(\mathrm{P}<0,01)$, na qual concluiu-se que consumir 1000mg de alhos processados em cápsulas durante 8 semanas pode significativamente reduzir a pressão arterial em pacientes hipertensos.

Stabler et al. (2012) relataram alguns estudos que utilizaram 200mg de pó de alho três vezes ao dia, no qual obteve-se uma redução significativa da pressão sistólica média e pressão diastólica, e que também obteve resultados positivos utilizando o pó do alho como terapia complementar a hidroclorotiazida- triantereno.

\section{Efeitos colaterais no uso do alho como fitoterápico}

No estudo de Ried et al. (2010), alguns participantes da pesquisa relataram algumas queixas quanto ao uso da cápsula de alho, a tolerabilidade da cápsula em geral foi elevada, apesar de um quarto $(24 \%)$ dos participantes relatarem eructação, refluxo, halitose, no entanto, estes efeitos foram considerados mínimos e descobriram formas de reduzi-los incluindo consumo de balas refrescantes, dividir a dosagem diária ou tomar as cápsulas com alimentos, e apenas 2 (8\%) participantes abandonaram o tratamento depois de dois meses por queixas gastrointestinais. 
Ried et al. (2013) também relataram em seu estudo que 4\% dos participantes (3 de 79) abandonaram o estudo depois de 4 semanas devido a queixas gastrointestinais. Outras queixas menores também foram relatadas pelos participantes, onde 32\% dos participantes queixaram-se de inchaço, flatulência e refluxo.

Nos estudos analisados por Xiong et al. (2015) os efeitos colaterais mais citados foram desconforto gástrico, eructação, refluxo, flatulência, prisão de ventre, diarreia, dor de cabeça e halitose, os participantes também relataram dificuldades para ingerir as cápsulas de alho.

\section{Utilização do alho na redução das morbidades e mortalidades}

O alho vem sendo considerado como uma forma de terapia alternativa no tratamento de hipertensão, visto que apresenta certa segurança e mínimos efeitos colaterais a quem o utiliza, porém ainda existe uma escassez de informação, o que dificulta a compreensão dos reais efeitos em potencial do alho em tratamentos clínicos (Xiong et al., 2015).

Nos estudos de Bahadoran et al. (2017) observou-se que o consumo de vegetais allium, tais como alho e cebola em uma quantidade significativa foram associados a um menor risco de hipertensão arterial e disfunções renais, reduzindo as chances de complicações cardiovasculares decorrentes da HAS.

O uso de extrato de alho envelhecido no tratamento de HAS não controlada também mostrou uma redução significativa nos valores da PA, tal redução pode ser comparada às obtidas com uso de anti-hipertensivos comumente prescritos. Assim, uma redução acerca de 10mmHg na PAS está diretamente associada a redução de risco de doenças cardiovasculares, sendo uma terapia alternativa, tolerada e altamente aceitável (Ried, Frank, \& Stocks, 2013).

Sobenin et al. (2009) afirmam que por ter um efeito hipotensor o alho assim como os derivados do mesmo grupo que o compõem (vegetais allium) podem contribuir estatisticamente de forma moderada para a redução e prevenção de doenças cardiovasculares, tendo em vista que a HAS é um dos principais riscos para o desenvolvimento dessas complicações. Porém, ainda não existe evidências suficientes que possam determinar o uso do alho como uma prática terapêutica com benefícios em relação a redução do risco de mortalidade e morbimortalidade cardiovasculares (Stabler, Tejani, Huynh, \& Fowkes, 2012).

\section{Os benefícios do allium vegetais na dieta}

A inserção de allium na dieta pode ter vários efeitos positivos no organismo, estudos sugerem que o alho pode ser eficaz na redução de colesterol e também na redução da PA em indivíduos hipertensos e saudáveis, por ter propriedades antioxidantes que potencializam os efeitos dos anti-hipertensivos (Bahadoran, Mirmiran, Momenan, \& Azizi, 2017).

Muitas vezes o tratamento com anti-hipertensivo não apresenta uma adesão adequada aos pacientes devido aos custos da medicação e de efeitos colaterais que podem ocorrer, em decorrência disso o alho vem sendo utilizado como uma terapia alternativa, não substituindo o uso dos anti-hipertensivos no controle da PA, pois é um método que ainda precisa ser objeto de diversos estudos para assim compreender seus efeitos na prática clínica (Wang et al., 2015).

Nos estudos de Ried et al. (2013) ao realizarem um ensaio utilizando extrato de alho envelhecido em pacientes com HAS, obteve-se uma redução nos níveis limítrofes da pressão arterial em pacientes que faziam uso de anti-hipertensivos regularmente e até naqueles que tinham HAS não controlada. Em pacientes com idade mais avançada tais efeitos não foram observados, o que sugere a influência de outros fatores, tais como genéticos e fisiológicos.

Acredita-se que os efeitos dos vegetais allium sobre a HAS podem variar devido a diferentes padrões e hábitos alimentares, portanto, a forma como são utilizados, a dosagem, os fatores ambientais, nutricionais, genéticos e até medicamentosos, estariam relacionados aos efeitos positivos ou negativos do uso de vegetais de allium no controle da hipertensão (Bahadoran et al., 2017). 


\section{Considerações Finais}

Este estudo permitiu descrever os benefícios do uso do extrato de alho no tratamento da hipertensão arterial de acordo com a Literatura, onde foram observados diversos efeitos positivos entre eles destaca-se redução do colesterol, redução da pressão arterial tanto em indivíduos hipertensos quanto em indivíduos saudáveis, e seu potencial positivo para auxiliar o tratamento convencional da hipertensão arterial.

Foi observado ainda que aumentar a ingestão de vegetais allium, pode estar associada a redução de triglicerídeos e depuração de creatinina, o que proporciona uma melhoria da função renal e diminuição da pressão arterial. Vale ressaltar ainda que o uso do alho apesar das baixíssimas contraindicações, pode ocasionar alguns efeitos colaterais em uma pequena parcela da população, são eles: distúrbios gastrointestinais, náuseas, refluxo, flatulência, prisão de ventre, diarreia ou até leve alteração no paladar.

Nesse aspecto, cabe aos profissionais da saúde avaliarem a melhor forma de introduzir o extrato de alho para auxiliar o tratamento da HAS, sempre respeitando as particularidades de cada cliente, monitorando o grau de conhecimento e aceitação dos indivíduos e observando se há presença de sinais de efeitos adversos.

Como limitações da pesquisa tem-se o fato de que há poucos estudos disponíveis que sejam capazes de investigar e comprovar todos os pontos positivos e negativos da utilização do extrato de alho no tratamento tanto da Hipertensão quanto no tratamento de outras patologias, elenca-se ainda o fato de que muitas vezes os profissionais da área da saúde preferem receitar somente fármacos e não utilizam nenhum tipo de terapia complementar.

Por fim, o estudo possibilitou expandir os conhecimentos acerca dos benefícios da utilização do extrato de alho como fitoterapia no controle da hipertensão. Mais estudos são necessários para explorar a eficácia de terapias adjuvantes com o uso do extrato de alho envelhecido; a associação entre a dose e a duração com a alteração da pressão arterial; a segurança do uso a longo prazo e o efeito do extrato de alho envelhecido sobre outros fatores, como o risco cardiovascular. Dessa forma, espera-se que novas pesquisas sejam realizadas nesta linha de investigação, a fim de provar a importância do uso do extrato de alho, bem como, outras terapias complementares para auxiliar no tratamento de diversas patologias.

\section{Referências}

Bahadoran, Z., Mirmiran, P., Momenan, A. A., \& Azizi, F. (2017). Allium vegetable intakes and the incidence of cardiovascular disease, hypertension, chronic kidney disease, and type 2 diabetes in adults: a longitudinal follow-up study. JournalofHypertension, 35(9), 1909-1916.

Bork, A. M. T. (2005). Enfermagem baseada em evidências: Guanabara Koogan.

Bueno, M. J. A., Martínez, B. B., \& Bueno, J. C. (2016). Manual de plantas medicinais e fitoterápicos: utilizados na cicatrização deferidas: Univás.

Chagas, F. C., Zanetti, J. F., \& Donatini, R. S. (2012). Allium sativum L. na prevenção e tratamento de doenças cardiovasculares. Rev. de Biologia e Farmácia, $7(2), 1-11$.

Fernández-Cervilla, A. B., Piris-Dorado, A. I., Cabrer-Vives, M. E., \& Barquero-González, A. (2013). Current status of Complementary Therapies in Spain in nursing degree. Revista Latino-americana de Enfermagem, 21(3), 679-686.

Ischkanian, P. C., \& Pelicioni, M. C. F. (2012). Challenges of complementary and alternative medicine in the SUS aiming to health promotion. Rev. bras. crescimento desenvolvimento humano, 22(2), 233-238.

Ministério da Saúde. (2015). Política nacional de práticas integrativas e complementares no SUS: atitude de ampliação de acesso (2a ed). Brasília.

Nobre, F., Coelho, E. B., Lopes, P. C., \& Geleilete, T. J. M. (2013). Essential Arterial Hypertension. Medicina, 46(3), $256-272$.

Oliveira, F. B. M., Costa, A. C. A. L., Alves, D. L., França, J. F., Macedo, M. S., \& Santos, R. D. (2016). Relação entre a sobrecarga de trabalho e erros de administração de medicação na assistência hospitalar. Revista Ciências \& Saberes, 2(2), 325-334.

Ried, k., Frank, O. R., \& Stocks, N. P. (2010). Aged garlic extract lowers blood pressure in patients with treated but uncontrolled hypertension: a randomised controlled trial. Maturitas, 67(2), 144-150.

Ried, k., Frank, O. R., \& Stocks, N. P. (2013). Aged garlic extract reduces blood pressure in hypertensives: a dose-response trial. European Journal of Clinical Nutrition, ;67(1), 64-70. 
Research, Society and Development, v. 10, n. 2, e7610212024, 2021

(CC BY 4.0) | ISSN 2525-3409 | DOI: http://dx.doi.org/10.33448/rsd-v10i2.12024

Silva, B. Q., \& Hahn, S. R. (2011). Uso de plantas medicinais por indivíduos com hipertensão arterial sistêmica, diabetes mellitus ou dislipidemias. Revista Brasileira de Farmácia Hospitalar e Serviços de Saúde, 2(3), 36-40.

Simons, S., Wollersheim, H., \& Thien, T. (2009). A systematic review on the influence of trial quality on the effect of garlic on blood pressure. Neth J Med, 67(6), 212-219.

Sobenin, I. A., Andrianova, I. V., Fomchenkov, I. V., Gorchakova, T. V., \& Orekhov, A. N. (2009). Time-released garlic powder tablets lower systolic and diastolic blood pressure in men with mild and moderate arterial hypertension. Hypertension Research, 32(6), 433-437.

Souza, M. T., Silva, M. D., \& Carvalho, R. (2010). Revisão integrativa: o que é e como fazer. Einstein, 8(1), 102-106.

Stabler, S. N., Tejani, A. M., Huynh, F., \& Fowkes, C. (2012). Garlic for the prevention of cardiovascular morbidity and mortality in hypertensive patients (Review). Cochrane Database of Systematic Reviews, (8), 1-25.

Wang, H.-P., Yang, J., Qin, L.-Q., \& Yang, X.-J. (2015). Effect of Garlic on Blood Pressure: A Meta-Analysis. The Journal of Clinical Hypertension, 17(3), 223-231.

Xiong, X. J., Wang, P. Q., Li, S. J., Li, X. K., Zhang, Y. Q., \& Wang, J. (2015). Garlic for hypertension: A systematic review and meta-analysis of randomized controlled trials. Phytomedicine, 22(3), 352-361. 